\title{
SIMULATION OF A LOW-REYNOLDS NUMBER FLOW AROUND A CYLINDER FOLLOWING A FIGURE-8-PATH
}

\author{
L. BARANYI
}

Department of Fluid and Heat Engineering, University of Miskolc

H-3515 Miskolc-Egyetemváros, Hungary; E-mail: arambl@uni-miskolc.hu

\begin{abstract}
This numerical study investigates a circular cylinder placed in a uniform stream and moving along a slender figure-8-path, using a 2D computational method based on the finite difference method. The effects of in-line amplitude of oscillation and of frequency ratio are investigated. Computations for varying amplitude values were carried out at $R e=150,200$ and 250 for a clockwise orbit (in the upper loop). Time-mean and rms values of force coefficients yielded smooth curves and tended to increase with amplitude.

The effect of frequency ratio was investigated at $\mathrm{Re}=200,250$ and 300 in the lock-in domain for both clockwise (CW) and anticlockwise (ACW) orientation. Results differ substantially depending on the direction of orientation. Mechanical energy transfer was always positive in ACW direction, which may lead to vortex-induced vibration, and always negative for CW orientation. The time-mean of drag was much lower for CW over the whole frequency ratio domain investigated. For the CW orbit vortex switches were found at specific frequency ratios at $\mathrm{Re}=250$ and 300. Limit cycle curves for the CW orbit before and after a jump were symmetric, mirror images, and quite complex, while vorticity contours were close to symmetry. These results indicate the possibility of symmetry-breaking bifurcation.
\end{abstract}

Keywords: circular cylinder, figure-8-path, forced oscillation, mechanical energy transfer, lock-in

\section{Introduction}

Flow around a single circular cylinder is very rich in physical phenomena, and this is even more so when the cylinder is oscillating. Some examples of these in real life are silos or smokestacks in wind or underwater pipes in a current. When vortices are shed from the structure a periodic force is generated, which might lead to the vibration of the structure, especially if the damping is small. The motion resulting from this force usually has either one or two degrees of freedom. The most frequently investigated type of one-degree-offreedom (1-DoF) cylinder motion is the transverse cylinder oscillation. Both experimental and numerical studies have dealt with pure transverse cylinder motion, e.g. Williamson and Roshko [1], Lu and Dalton [2] and Blackburn and Henderson [3]. Less often investigated is pure in-line cylinder motion, e.g., Cetiner and Rockwell [4], Al-Mdallal et al. [5] and Mureithi et al. [6].

However, far fewer investigations have been carried out for combined, two-degree-of-freedom (2-DoF) cylinder motion. In reality, however, both motions are often present, leading to a Lissajous-type path. The vortex-induced vibration (VIV) arising in such cases can lead to problems such as fatigue and damage of structures.

Kheirkhah and Yarusevych [7] suggest that where the mass ratio (the ratio of the mass of the vibrating system to the mass of the displaced fluid) is high, as mainly occurs when a structure is oscillating in air, then the frequency of oscillation in in-line and in transverse directions are approximately equal to each other. This can lead to an elliptical path, such as that observed in tube bundles in heat exchangers [8]. Elliptical cylinder motion is studied in [7, 9-11], among others. The occurrence of vortex switches at certain frequency or amplitude values has been noted in [2] and [10].

Numerical studies often place a cylinder in forced motion in order to gain an approximation of fluid-structure interaction. This results in a simplified model. Although, as Williamson states in [12], a direct relationship between free and forced vibration is 
difficult to confirm, it is a convenient approach to begin investigation of the complex phenomena involved when a bluff body is moving in flow.

A more typical Lissajous-type path, on the other hand, occurs with a low mass ratio, typically found when a cylinder is moving in liquid: cables and pipes submersed in flowing water may undergo fatigue or damage due to this kind of motion. In this case the frequency of the cylinder motion in in-line direction is approximately twice its frequency in transverse direction, as found for example in Jeon and Gharib [13], Jauvtis and Williamson [14] and Sanchis et al. [15]. The phase angle difference between in-line and transverse motion $\Theta$ results in different cylinder paths [13]. The experimental investigation of 2-DoF forced cylinder motion in [13] emphasises the importance of the phase difference between the two motions. They suggest that the phase value tends to vary within 45 degrees of zero for free vibration cases. Baranyi [16] in his numerical study examined the effect of phase angle difference between 0 and 45 degrees as suggested in [13] and found that $\Theta$ has a strong influence on force coefficients and mechanical energy transfer between the fluid and the cylinder. One typical Lissajous curve is a figure-eight-shaped path, while a path in the shape of an arc, or C-shape, has also been observed by Sanchis et al. in their experimental study for an elastically supported cylinder in the Reynolds number domain of $\operatorname{Re}=(1.3$ to 1.9$) \cdot 10^{4}$ [15] as well as in the numerical work of Prasanth and Mittal [17].

Perdikaris et al. [18] numerically investigated flow around a mechanically oscillated cylinder following a figure-8-path at Reynolds number $\mathrm{Re}=400$ while varying the transverse amplitude of oscillation. Their study looked at the power transfer parameter for two frequency ratios of 0.5 and 1 . They found that the orientation of the motion (clockwise or anticlockwise orbit in the upper loop) influences the results, generally leading to higher force coefficients and power transfer for the anticlockwise orientation. Peppa et al. [19] carried out a similar investigation for $\mathrm{Re}=400$ at frequency ratios of $0.9,1$ and 1.1 at two different $A_{x} / A_{y}$ ratio values. They also carried out computations for both directions of orbit and found that an anticlockwise orbit in the upper loop resulted in a positive power coefficient, meaning an increased chance of vortexinduced vibration for a cylinder in free vibration.

An earlier numerical study of mine [20] dealt with forced figure-8-motion for $\mathrm{Re}=150,200$ and 250 against in-line oscillation amplitude and $\mathrm{Re}=250$ for frequency ratio, for clockwise orientation only. Force coefficients and mechanical energy transfer were investigated against both in-line oscillation amplitude and frequency ratio. No sudden change in vortex structure was detected when investigated against amplitude, but a vortex switch was found against the fre- quency ratio. The current numerical investigation introduces further computational results for the frequency ratio and also investigates figure-8-motion with an anti-clockwise orientation (at $\operatorname{Re}=200,250$ and 300).

\section{Nomenclature:}

$A_{x, y} \quad$ amplitude of oscillation in $x$ or $y$ directions, respectively, non-dimensionalised by $d$

$C_{D} \quad$ drag coefficient, $2 F_{D} /\left(\rho U^{2} d\right)$

$C_{L} \quad$ lift coefficient, $2 F_{L} /\left(\rho U^{2} d\right)$

$d \quad$ cylinder diameter $(\mathrm{m})$

$E$ mechanical energy transfer, non-dimensionalised by $\rho U^{2} d^{2} / 2$

$\mathbf{F} \quad$ force per unit length of cylinder, $F_{D} \mathbf{i}+F_{L} \mathbf{j}$ $(\mathrm{N} / \mathrm{m})$

$F_{D} \quad$ drag per unit length of cylinder $(\mathrm{N} / \mathrm{m})$

$F_{L} \quad$ lift per unit length of cylinder (N/m)

$f \quad$ oscillation frequency, non-dimensionalised by $U / d$

$\mathbf{i}, \mathbf{j} \quad$ unit vectors in $x$ and $y$ directions, respectively

$p \quad$ pressure, non-dimensionalised by $\rho U^{2}$

Re Reynolds number, $U d / v$

$R \quad$ radius, non-dimensionalised by $d$

St non-dimensional vortex shedding frequency

$T$ motion period, non-dimensionalised by $d / U$

$t \quad$ time, non-dimensionalised by $d / U$

tq torque coefficient, non-dimensionalised by $\rho U^{2} d^{2}$

$U$ free stream velocity, velocity scale $(\mathrm{m} / \mathrm{s})$

$\mathbf{v}_{0} \quad$ cylinder velocity, non-dimensionalised by $U$, $v_{0 x} \mathbf{i}+v_{0 y} \mathbf{j}$

$x, y \quad$ Cartesian co-ordinates, non-dimensionalised by $d$

$v \quad$ kinematic viscosity $\left(\mathrm{m}^{2} / \mathrm{s}\right)$

$\Theta \quad$ phase angle difference between transverse and in-line cylinder motion

$\rho \quad$ fluid density $\left(\mathrm{kg} / \mathrm{m}^{3}\right)$

Subscripts:

$\mathrm{fb} \quad$ fixed-body

$D \quad$ drag

$L \quad$ lift

$r m s$ root-mean-square value

$x, y \quad$ components in $x$ and $y$ directions

0 for cylinder motion; for stationary cylinder at the same $\mathrm{Re}$

\section{Computational method}

A non-inertial system fixed to the cylinder is used to compute two-dimensional (2D) low-Reynolds number unsteady flow around a circular cylinder placed in a uniform stream and forced to oscillate in transverse or in in-line direction, or in both. The non-dimensional Navier-Stokes equations for incompressible constantproperty Newtonian fluid, the equation of continuity 
and the Poisson equation for pressure can be written as follows:

$$
\begin{gathered}
\frac{\partial u}{\partial t}+u \frac{\partial u}{\partial x}+v \frac{\partial u}{\partial y}=-\frac{\partial p}{\partial x}+\frac{1}{\operatorname{Re}} \nabla^{2} u-a_{0 x} \\
\frac{\partial v}{\partial t}+u \frac{\partial v}{\partial x}+v \frac{\partial v}{\partial y}=-\frac{\partial p}{\partial y}+\frac{1}{\operatorname{Re}} \nabla^{2} v-a_{0 y} \\
D=\frac{\partial u}{\partial x}+\frac{\partial v}{\partial y}=0 \\
\frac{\partial^{2} p}{\partial x^{2}}+\frac{\partial^{2} p}{\partial y^{2}}=2\left[\frac{\partial u}{\partial x} \frac{\partial v}{\partial y}-\frac{\partial u}{\partial y} \frac{\partial v}{\partial x}\right]-\frac{\partial D}{\partial t}
\end{gathered}
$$

In these equations, $u$ and $v$ are the $x$ and $y$ components of velocity, $t$ is time, $p$ is the pressure, Re is the Reynolds number based on cylinder diameter $d$, free stream velocity $U$, and kinematic viscosity $v$, and $D$ is the dilation. Although $D$ is theoretically equal to 0 from Eq. (3), it is kept in Eq. (4) to avoid accumulation of numerical errors, following Harlow and Welch [21]. In Eqs. (1) and (2) $a_{0 x}$ and $a_{0 y}$ are the $x$ and $y$ components of cylinder acceleration, respectively.

On the cylinder surface, no-slip boundary condition is used for the velocity and a Neumann-type boundary condition is used for the pressure. At the far region, potential flow is assumed, which is reasonable aside from the narrow wake. Since the outer boundary of the computational domain is very far from the cylinder, this assumption results in only a small distortion of the velocity field, and only near the outer boundary wake region, see Baranyi and Shirakashi [22].

Boundary-fitted coordinates are used to impose the boundary conditions accurately. Using unique, single-valued functions, the physical domain bounded by two concentric circles is mapped into a rectangular computational domain with equidistant spacing in both directions (Fig. 1). In the physical domain logarithmically spaced radial cells are used, providing a fine grid scale near the cylinder wall and a coarse grid in the far field. The transformed governing equations and boundary conditions are solved by the finite difference method. Space derivatives are approximated by fourth order central differences, except for the convective terms for which a third-order modified upwind scheme is used. The Poisson equation for pressure is solved by the successive over-relaxation (SOR) method. The Navier-Stokes equations are integrated explicitly and continuity is satisfied at every time step. For further details see [10, 23].

The 2D code developed by the author has been extensively tested against experimental and computational results for a stationary cylinder, see e.g. [10, 24, 25], and computational results for cylinders oscillating in transverse or in in-line directions or following a circular path, including [2, 5, 9], with good agreement being found [10]. Comparisons with secondorder Runge-Kutta discretisation results showed that - at this small time step - the results agree well with each other [26], thus justifying the use of a first-order approach here. In this study the dimensionless time step is 0.0005 , the number of grid points is $481 \times 451$ (peripheral $\times$ radial), and a relatively large physical domain of $R_{2} / R_{1}=360$ has been chosen to enhance accuracy.

\section{Computational setup}

This study investigates the behaviour of flow past a cylinder placed in a uniform stream with its axis perpendicular to the velocity vector of the main flow. The cylinder is oscillated mechanically in both in-line and transverse directions in relation to the uniform stream. The motion of the centre of the cylinder is described by the following equations:
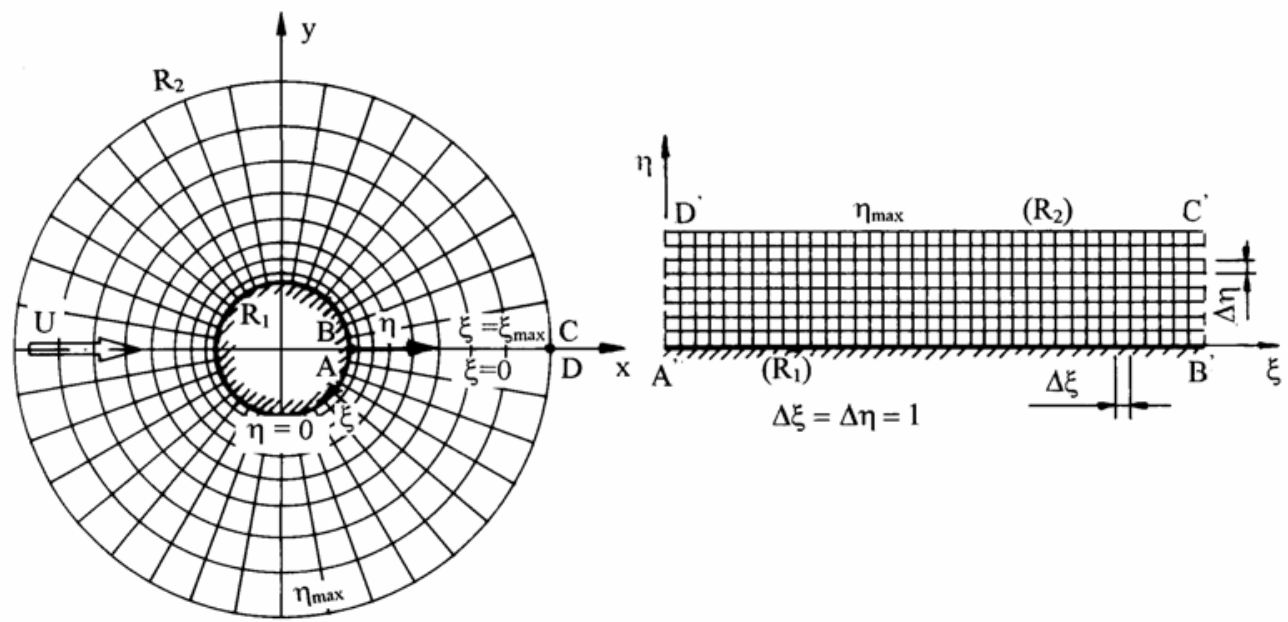

Fig. 1. Physical and computational domains 


$$
\begin{aligned}
& x_{0}=A_{x} \sin \left(4 \pi f_{y} t+\Theta\right), \\
& y_{0}=A_{y} \sin \left(2 \pi f_{y} t\right),
\end{aligned}
$$

where $\Theta$ is the phase angle difference between the two oscillations. Naturally the second time derivatives of $x_{0}$ and $y_{0}$ give the accelerations $a_{0 x}$ and $a_{0 y}$ in Eqs. (1) and (2).

The time-history of force coefficients (lift, drag, base pressure and torque), pressure and velocity fields are computed. From these data, time-mean (TM) and root-mean-square (rms) values of force coefficients, streamlines, and vorticity contours can be obtained.

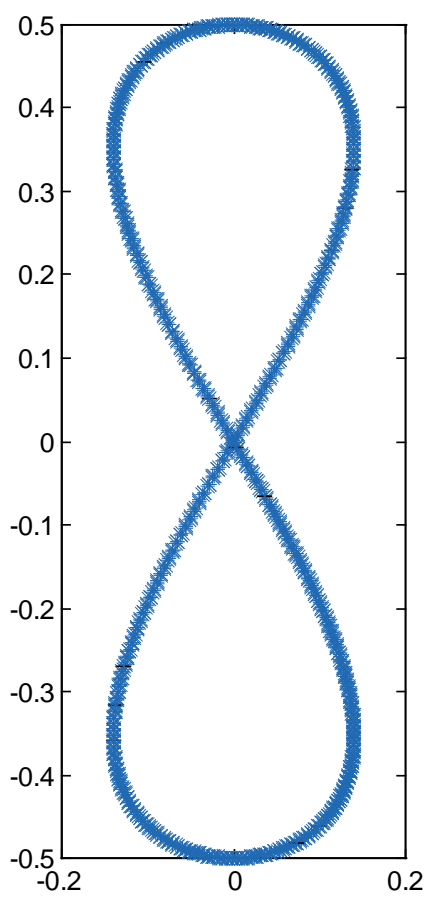

Fig. 2. The layout for the figure-8-path

Throughout this paper the lift and drag coefficients used, unless otherwise stated, do not contain the inertial forces originating from the non-inertial system fixed to the accelerating cylinder. Coefficients obtained by removing the inertial forces are often termed 'fixed body' coefficients (see [2]). The relationship between the two sets of coefficients can be written as

$$
\begin{aligned}
& C_{D}=C_{D f b}+\pi a_{0 x} / 2, \\
& C_{L}=C_{L f b}+\pi a_{0 y} / 2,
\end{aligned}
$$

where subscript fb refers to the fixed-body (understood in an inertial system fixed to the stationary cylinder) [27], and $a_{0 \mathrm{x}}$ and $a_{0 \mathrm{y}}$ denote the acceleration of the cylinder in $x$ and $y$ directions, respectively. Since the inertial terms are $T$-periodic functions, their time- mean values vanish, resulting in identical TM values for lift and drag in the inertial and non-inertial systems. Naturally the rms values of $C_{L}$ and $C_{D}$ will be somewhat different in the two systems (but this does not affect the curve being continuous).

The investigation was restricted to lock-in cases. Lock-in - the synchronisation between vortex shedding and cylinder motion - produces a periodic solution for each of the force coefficients. In this paper, we consider lock-in to be when the vortex shedding frequency is identical to $f_{y}$, the frequency of transverse cylinder oscillation.

To create a figure-8-path with a clockwise (CW) and anticlockwise (ACW) orientation in the upper loop, respectively, the conditions

$$
\begin{array}{lll}
f_{x}=2 f_{y} ; & \Theta=\pi & \text { (for CW), } \\
f_{x}=2 f_{y} ; & \Theta=0 & \text { (for } \mathrm{ACW})
\end{array}
$$

should be fulfilled in Eqs. (5) and (6).

The non-dimensional energy transfer $E$ originally introduced in [3] for a transversely oscillated cylinder was extended for a general two-degree-of-freedom (2-DoF) motion of the cylinder in [10]:

$$
E=\frac{2}{\rho U^{2} d^{2}} \int_{0}^{T} \mathbf{F} \cdot \mathbf{v}_{0} \mathrm{~d} t=\int_{0}^{T}\left(C_{D} v_{0 x}+C_{L} v_{0 y}\right) \mathrm{d} t,
$$

where $T$ is the motion period, $v_{0 x}$ and $v_{0 y}$ are the velocity of the cylinder in $x$ and $y$ directions, respectively. Since the frequencies in the two directions are different (see Eqs. (9) and (10)) the larger period of $T=$ $T_{y}=1 / f_{y}$ is chosen for the investigation. Equation (11) is also valid for 1-DoF cylinder motions; i.e., for pure transverse $\left(v_{0 x}=0\right)$ and for in-line $\left(v_{0 y}=0\right)$ cylinder motions. Some researchers (e.g. [18]) prefer to use the concept of non-dimensional power transfer instead of mechanical energy transfer $E$.

\section{Results}

For a single computation, all parameters $\left(A_{x}, A_{y}\right.$, Re, $f_{x}$, $\left.f_{y}, \Theta\right)$ are kept constant. The computations are then repeated at different $A_{x}$ (see Section 4.1.) or $f_{y} / \mathrm{St}_{0}$ (see Section 4.2) values to investigate the effect of oscillation amplitude and frequency ratio, respectively. Here $\mathrm{St}_{0}$ is the non-dimensional vortex shedding frequency from a stationary cylinder at the given Reynolds number. Only the locked-in domain was considered. For both cases, time-mean (TM) and root-mean-square (rms) values of lift $\left(C_{L}\right)$, drag $\left(C_{D}\right)$, and torque (tq) coefficients, furthermore the mechanical energy transfer $E$ between the cylinder and fluid were determined and plotted against the independent variable. 
Jumps representing vortex switches were found for the clockwise (CW) case at $\mathrm{Re}=250$ and 300 when the independent variable was the frequency ratio. Preand post-jump analyses were carried out in the vicinity of the jumps (see Section 4.3.).
4.1. Effect of in-line amplitude for clockwise orbit in the upper loop

Here, three Re values were investigated: $\operatorname{Re}=150$, 200 , and 250. The frequency of transverse oscilla-

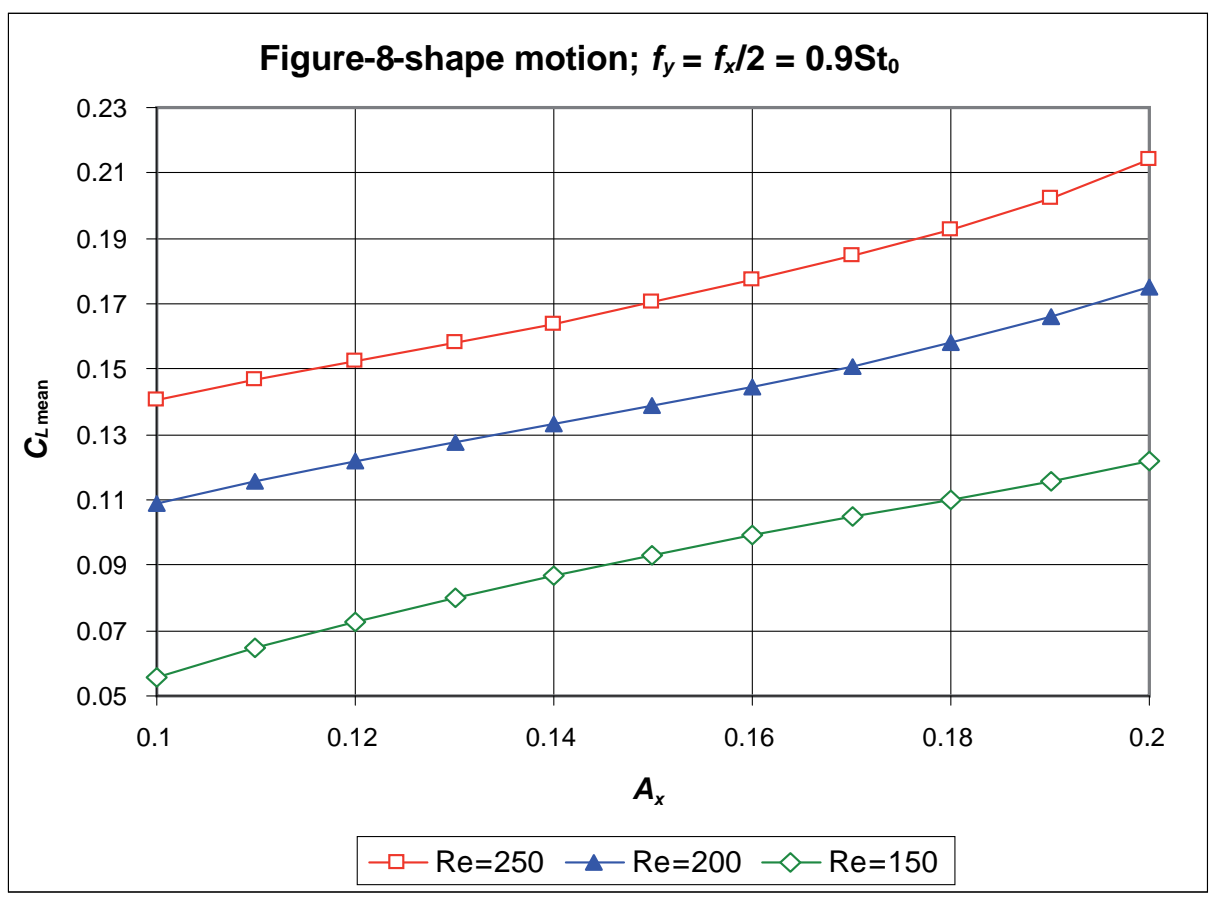

Fig. 3. Time-mean value of lift vs. $A_{x}$ for $\mathrm{Re}=150,200$ and 250

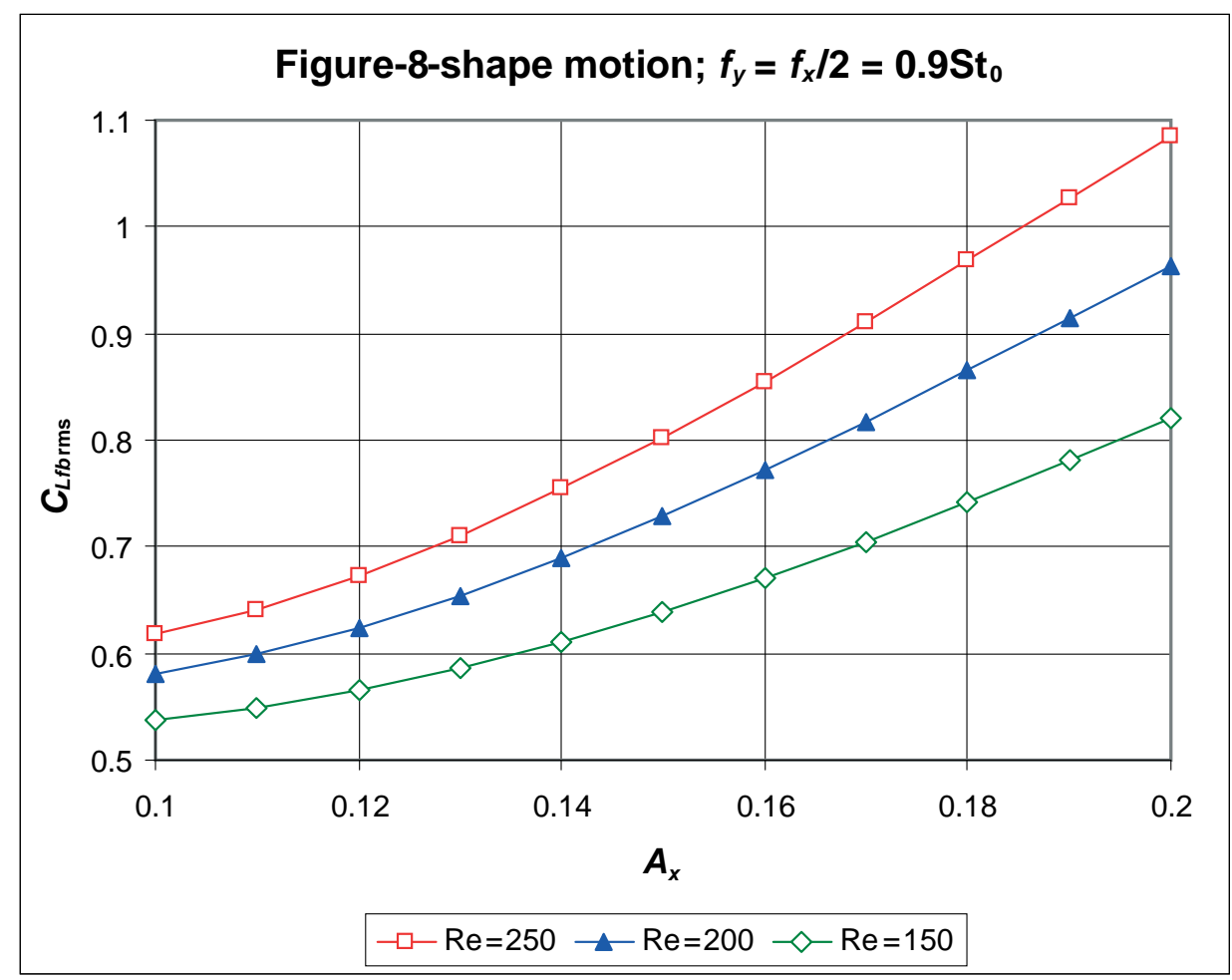

Fig. 4. Rms value of fixed body lift vs. $A_{x}$ for $\mathrm{Re}=150,200$ and 250 
tion was kept at $f_{y} / \mathrm{St}_{0}=0.9$, under resonant forcing. This frequency ratio ensures moderate amplitude values for subharmonic lock-in. The dimensionless transverse amplitude of oscillation $A_{y}$ was fixed at the value of 0.5 while $A_{x}$ was varied systematically between 0.1 and 0.2 to keep the figure-8-path slender found experimentally, e.g. in [15].

Figure 3 shows the variation of TM of lift against $A_{x}$ for the three different Re values. As seen in the figure, all TM values of lift in the domain are posi-

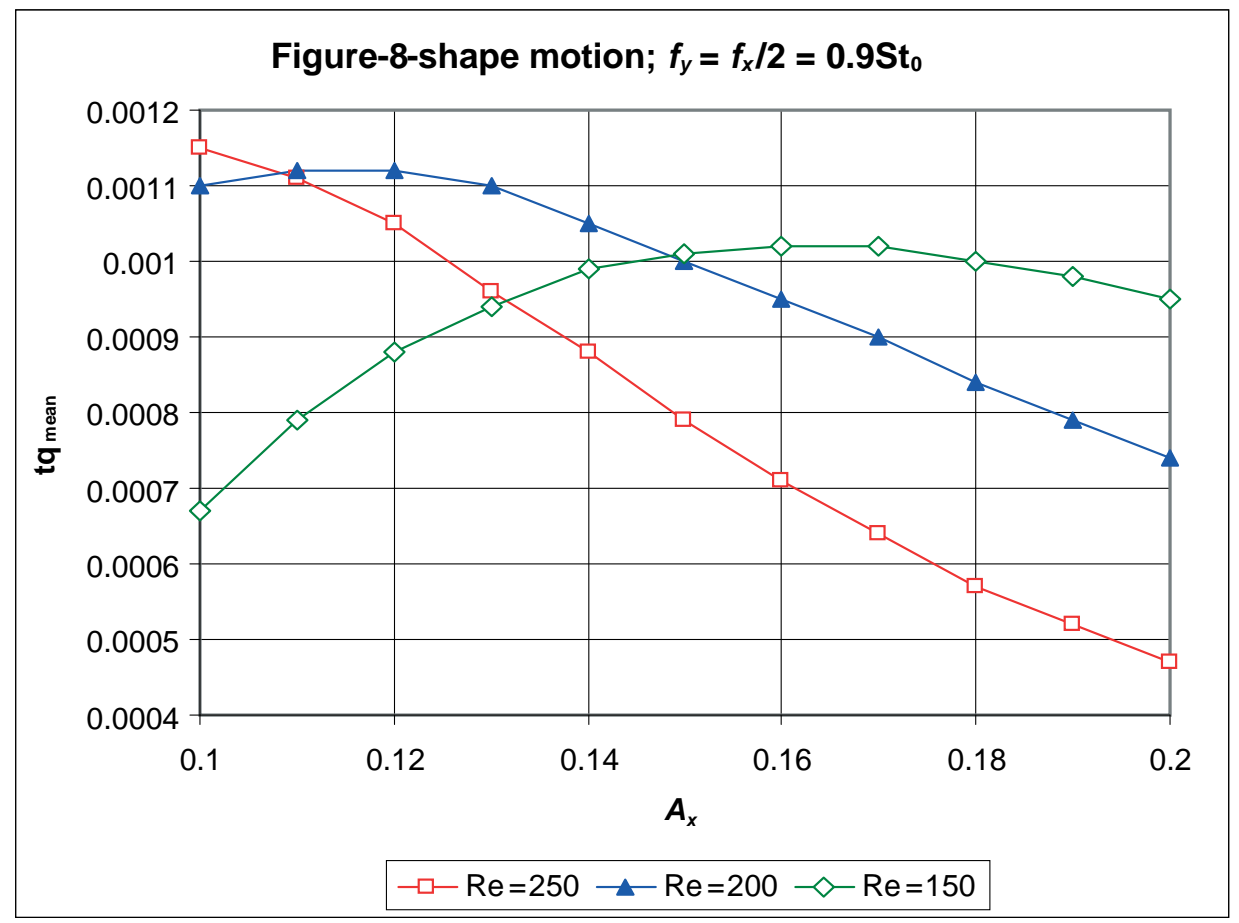

Fig. 5. Time-mean value of torque vs. $A_{x}$ for $\mathrm{Re}=150,200$ and 250

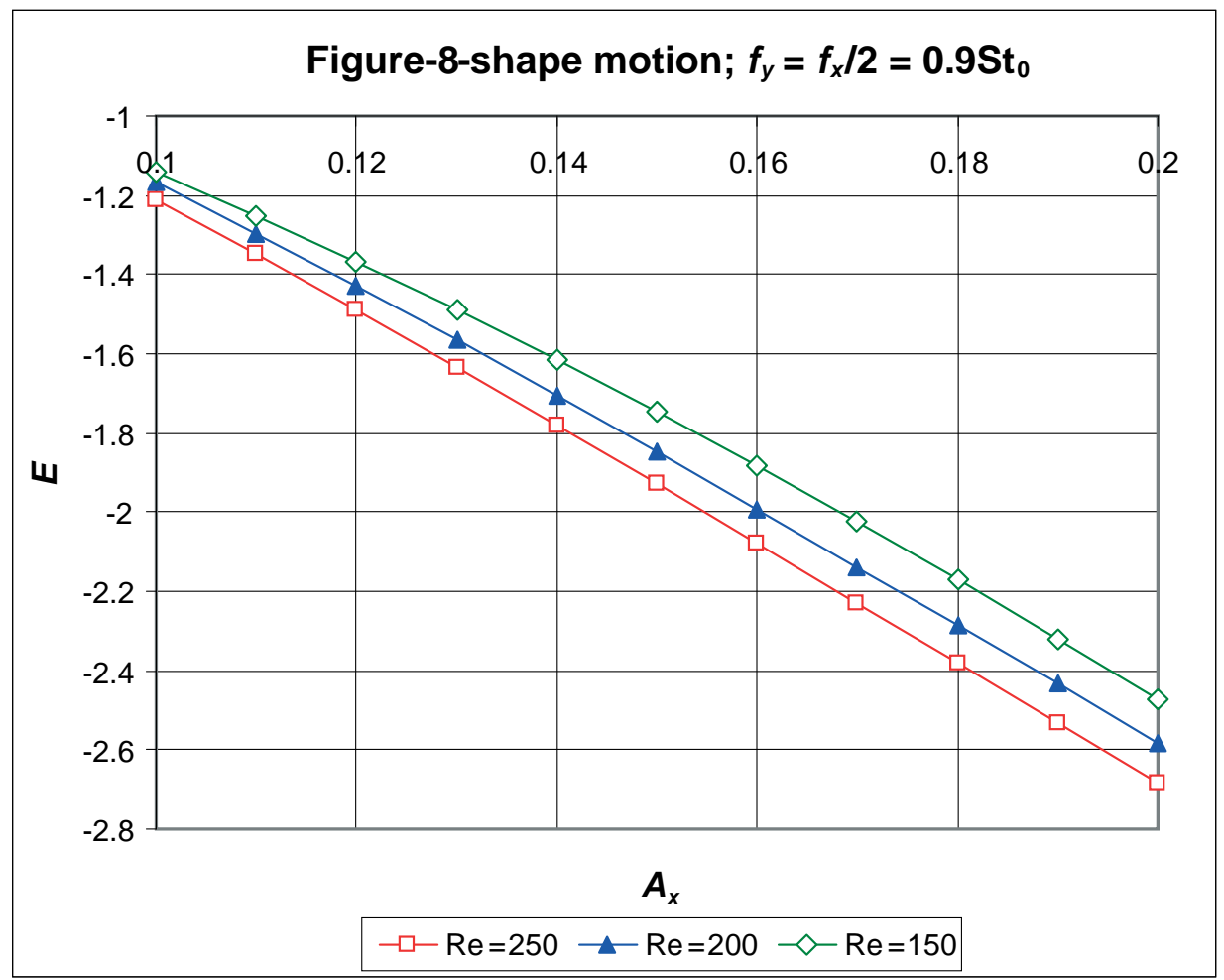

Fig. 6. Mechanical energy transfer $E$ vs. $A_{x}$ for $\mathrm{Re}=150,200$ and 250 
tive, the values increase with both $A_{x}$ and Reynolds number, and there are no jumps in the values. Please note that the TM of lift is zero for a stationary cylinder or for a cylinder oscillating transverse to the main stream but for several other types of cylinder motion it is not $[10,28]$.

Figure 4 shows the variation of rms value of the fixed body (see Eqs. (7) and (8)) lift coefficient against $A_{x}$ for the three Re values. The trend for these curves is very similar to that shown in Fig. 3. Other TM and rms curves show the same trend, with the exception of the TM of the torque coefficient (non-dimensional torque due to the shear stress on the cylinder surface), shown in Fig. 5, where no general tendency can be ascertained yet. These results are unexpected, since in earlier investigations it has generally been found that the time-mean of the torque coefficient behaves similarly to the lift coefficient; see e.g. [28-30]. Further investigations are needed in this area.

Finally, Fig. 6 shows the variation of $E$ with $A_{x}$ for the three Reynolds numbers. This mechanical energy transfer was negative for all of the investigated cases, meaning that energy is transferred from the cylinder to the fluid. The absolute value of $E$ increases with increasing amplitude $A_{x}$ and also with Re. As it can be seen in the figure, the relationship between $E$ and $A_{x}$ is almost linear for all three Reynolds numbers. Since all mechanical energy transfer values are negative, there is no danger of vortex-induced vibration (VIV) for free vibration cases.
Note that no jumps indicating vortex switches were found within this parameter domain, in contrast to findings for an orbiting cylinder [10] or cylinder moving in-line [28], meaning that no bifurcation was found within this domain. For this reason, it was decided not to extend the investigation against amplitude $A_{x}$ for any further Reynolds numbers. This is also why the investigation against amplitude $A_{x}$ for anticlockwise orientation was ignored at this stage of the research (to be frank, results plotted against frequency ratio proved to be more intriguing).

\subsection{Effect of frequency ratio for both directions of orbit}

Here, three Reynolds numbers were investigated, $\mathrm{Re}=$ 200, 250 and 300. $A_{y}$ was chosen to be $0.5 ; A_{x}$ was 0.14 during the whole investigation. This choice ensures a slender cylinder path. The usual step between two consecutive frequency ratio $f_{y} / \mathrm{St}_{0}$ values was 0.01 , except around the area of the jump, where computations were carried out at smaller intervals to determine the location of the jump. The investigations were limited to locked-in cases, i.e. when the vortex shedding synchronises with the cylinder motion (vortex shedding frequency is equal to $f_{y}$ ).

Figure 7 shows the TM of lift $C_{L}$ for six cases: three Reynolds numbers for both clockwise (CW) and anticlockwise (ACW) directions of orbit in the upper loop of figure-8-path. It can be seen in the figure that

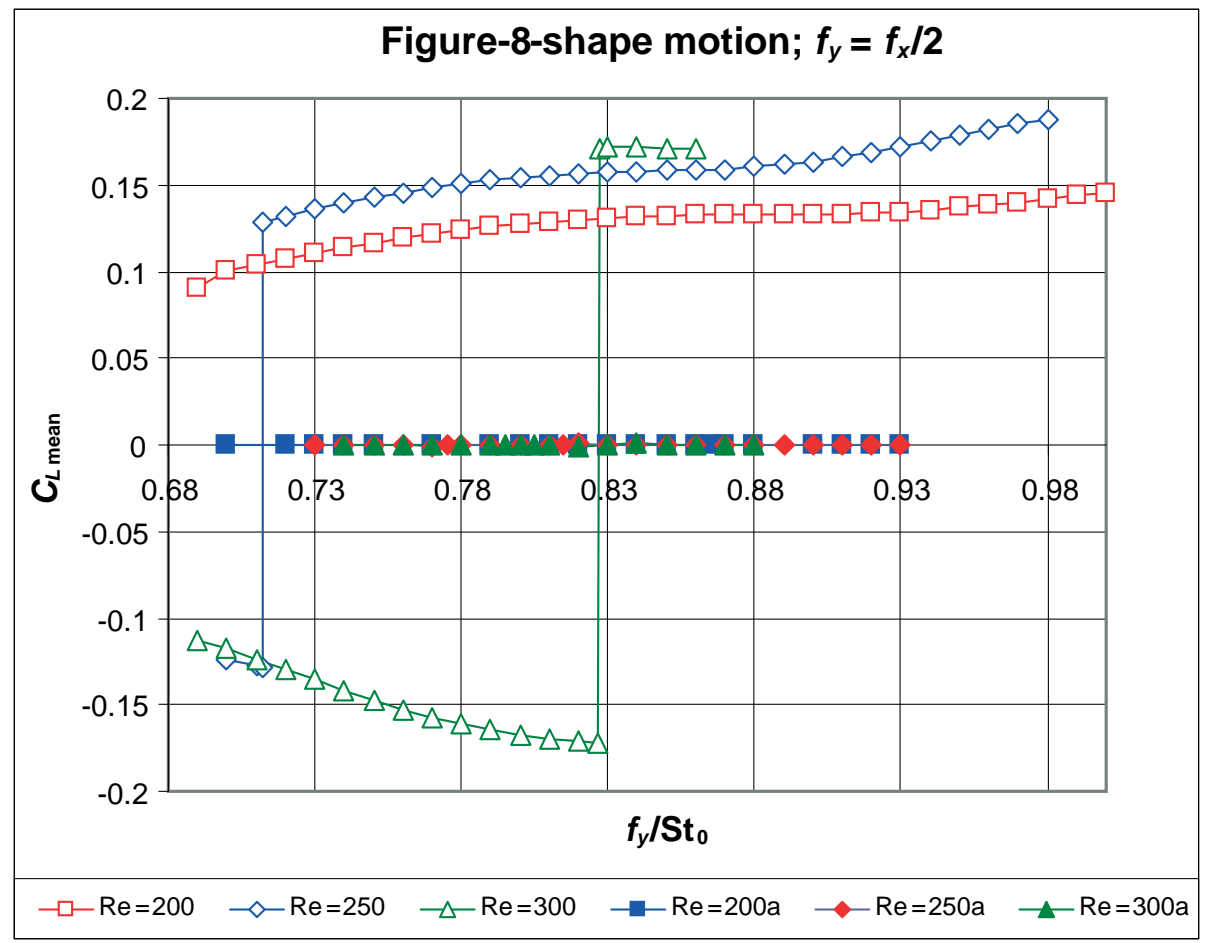

Fig. 7. Time-mean values of lift coefficient vs. frequency ratio for clockwise $(\mathrm{Re}=200,250,300)$ and anticlockwise $(\mathrm{Re}=200 \mathrm{a}, 250 \mathrm{a}, 300 \mathrm{a})$ orbit in the upper loop 
the TM values of lift for the ACW case (filled signals) are zero for all three Reynolds numbers. Similar results were found for pure transverse cylinder oscillation in [29], thus demonstrating one kind of symme- try, as investigated by Crawford and Knobloch [31]. The curves for the CW cases (empty signals) show one jump each for $\mathrm{Re}=250$ and 300, representing a sudden switch in vortex structure (no jump was found

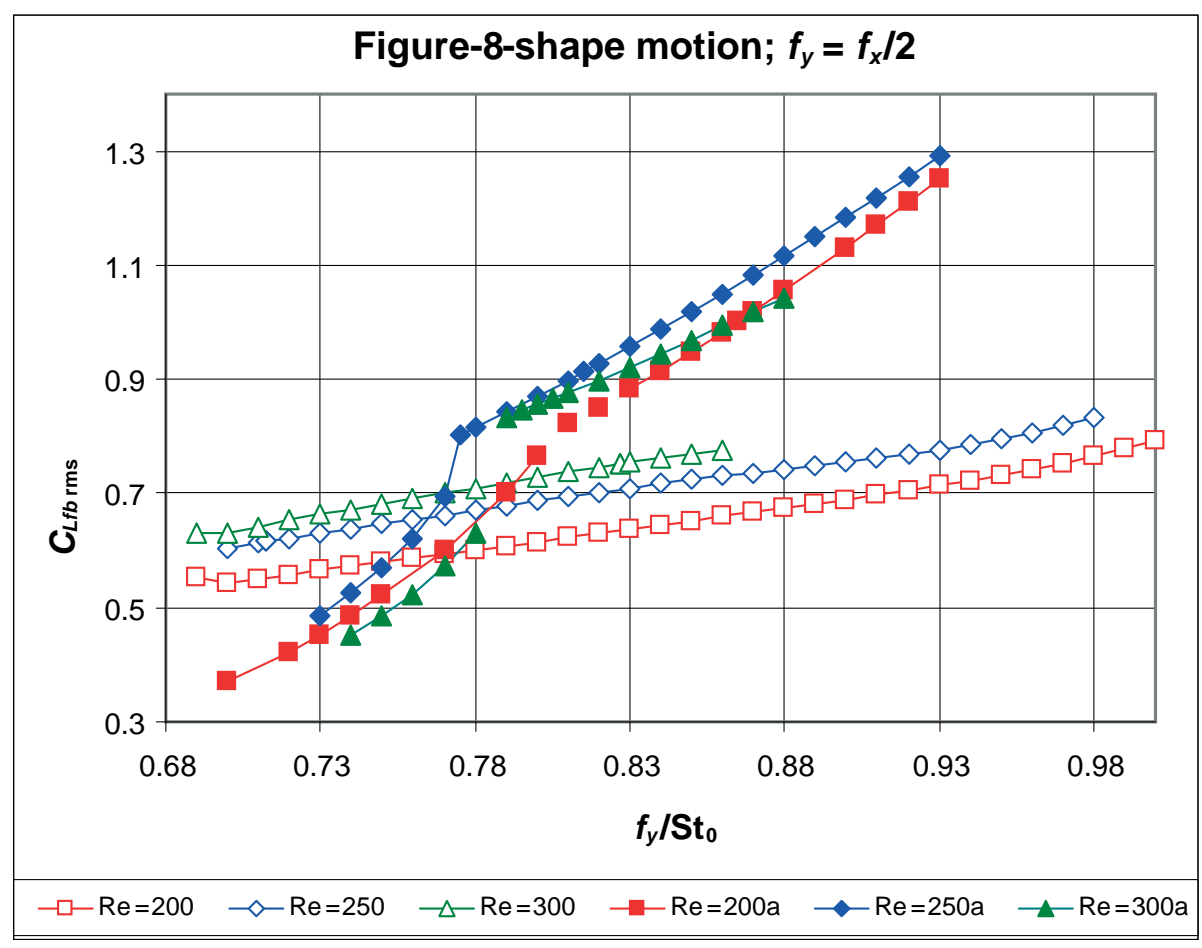

Fig. 8. Rms values of fixed-body lift vs. frequency ratio for clockwise $(\operatorname{Re}=200,250,300)$ and anticlockwise ( $\operatorname{Re}=200 a, 250 a, 300 a)$ orbit in the upper loop

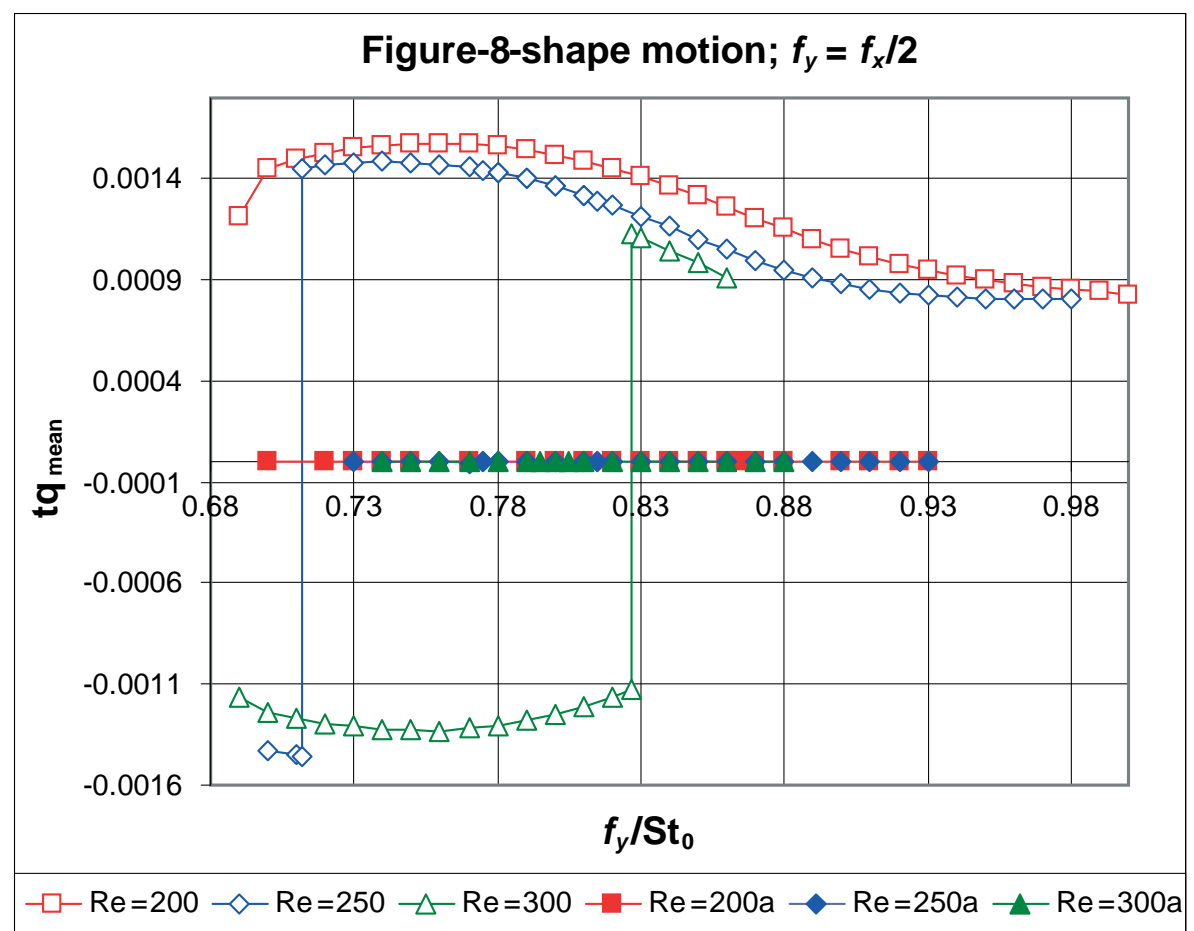

Fig. 9. Time-mean values of torque coefficient vs. frequency ratio for clockwise $(\mathrm{Re}=200,250$, $300)$ and anticlockwise $(\mathrm{Re}=200 \mathrm{a}, 250 \mathrm{a}, 300 \mathrm{a})$ orbit in the upper loop 


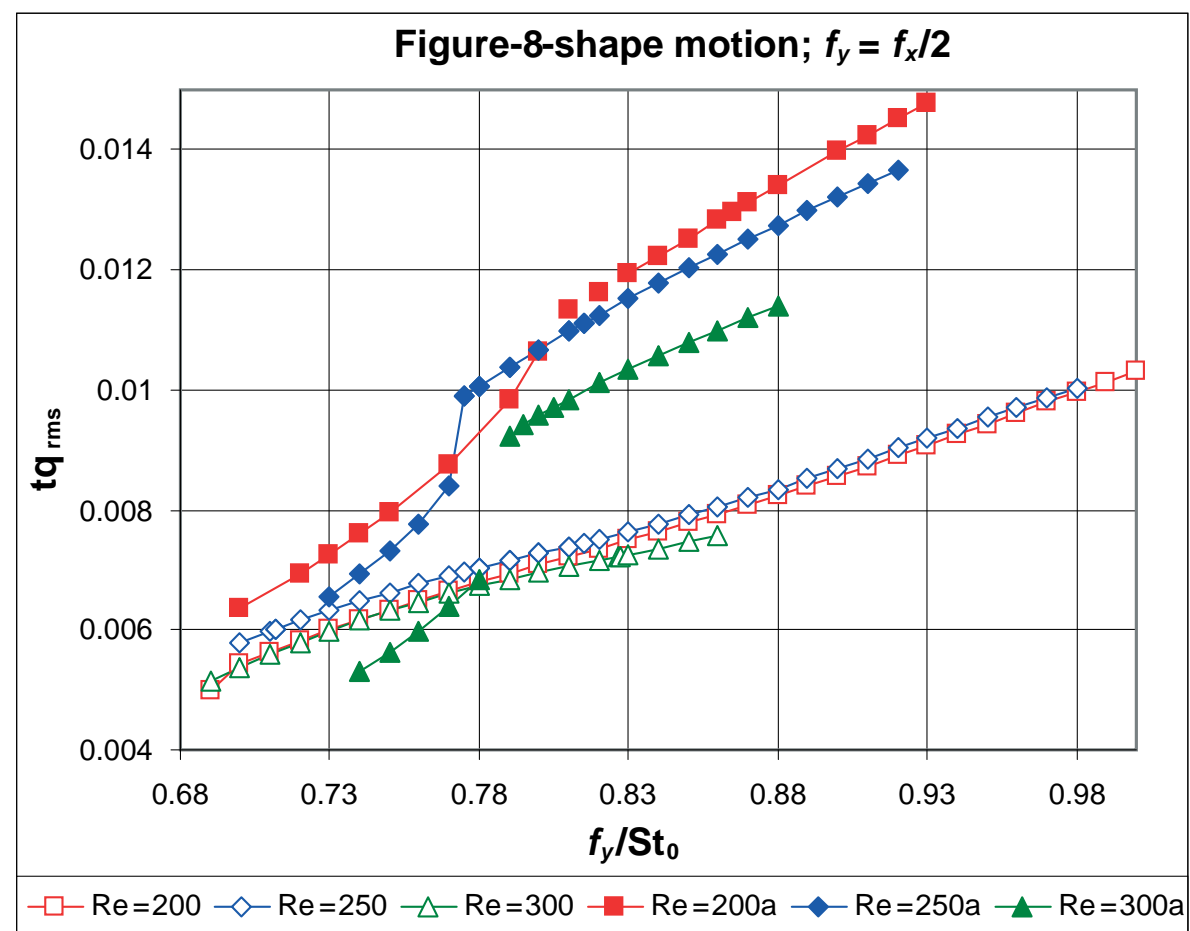

Fig. 10. Rms values of torque coefficient vs. frequency ratio for clockwise $(\operatorname{Re}=200,250,300)$ and anticlockwise $(\operatorname{Re}=200 \mathrm{a}, 250 \mathrm{a}, 300 \mathrm{a})$ orbit in the upper loop

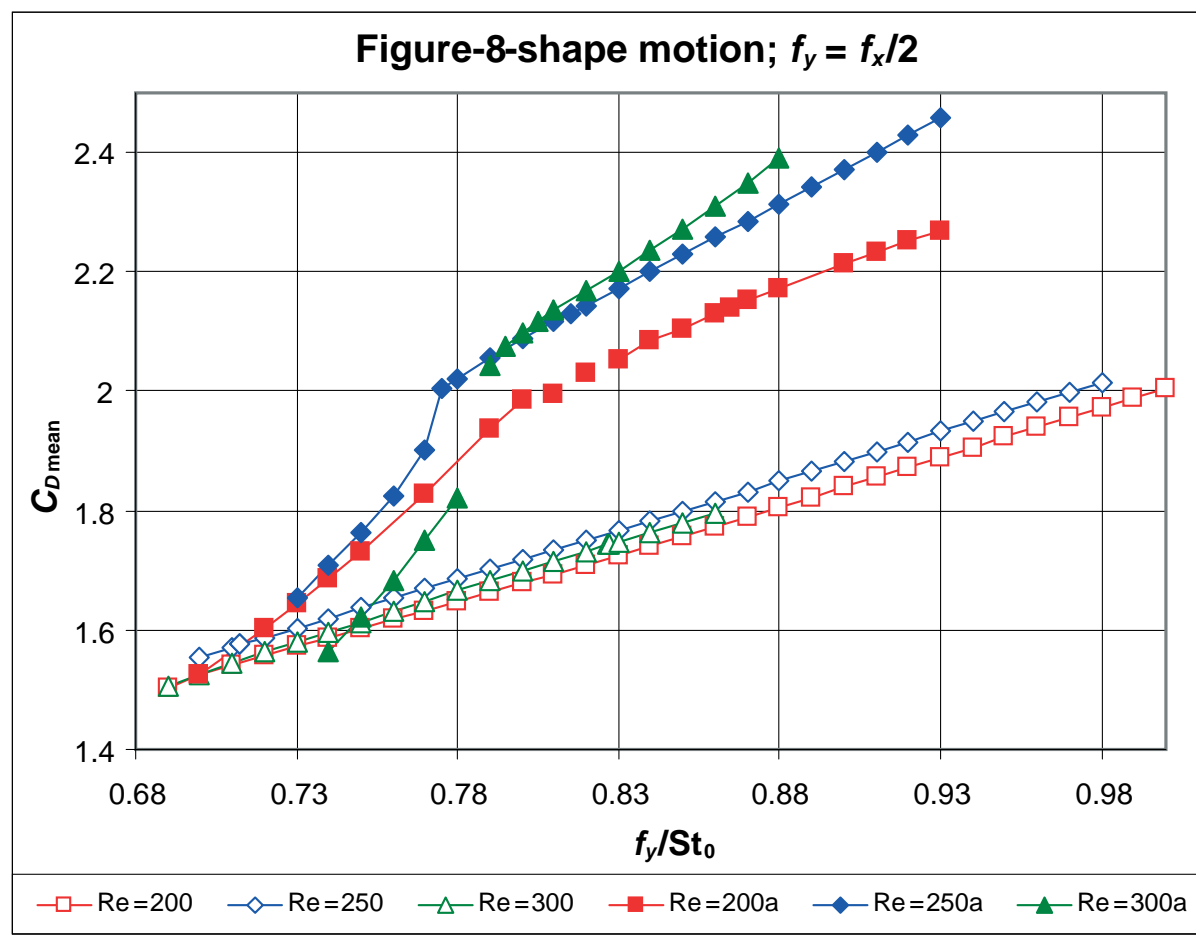

Fig. 11. Time-mean values of drag coefficient vs. frequency ratio for clockwise $(\operatorname{Re}=200,250$, $300)$ and anticlockwise $(\mathrm{Re}=200 \mathrm{a}, 250 \mathrm{a}, 300 \mathrm{a})$ orbit in the upper loop

for $\mathrm{Re}=200$ in the lock-in domain). The values jump from negative to positive values, and their absolute values are approximately equal to each other. Considering findings from earlier studies (e.g., [10, 28]), there exist two so-called state curves that can be repro- duced by varying some parameters or the initial conditions. These state curves were found to be symmetric around zero (i.e., the value for a stationary cylinder) for in-line oscillation [28], and this may be the case here, as well. If so, the vortex switch - the jump in the 


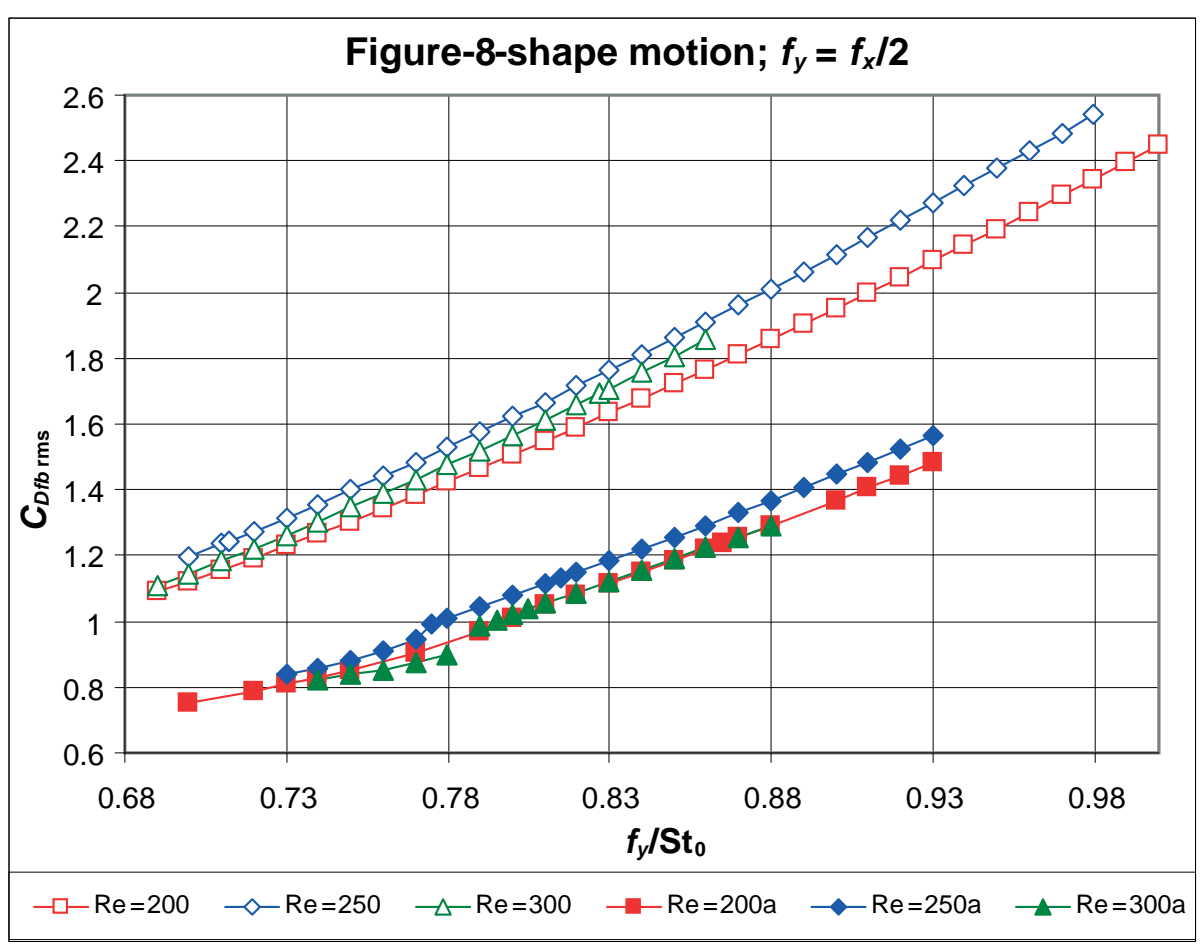

Fig. 12. Rms values of fixed-body drag coefficient vs. frequency ratio for clockwise $(R e=200,250$, $300)$ and anticlockwise $(\operatorname{Re}=200 a, 250 a, 300 a)$ orbit in the upper loop

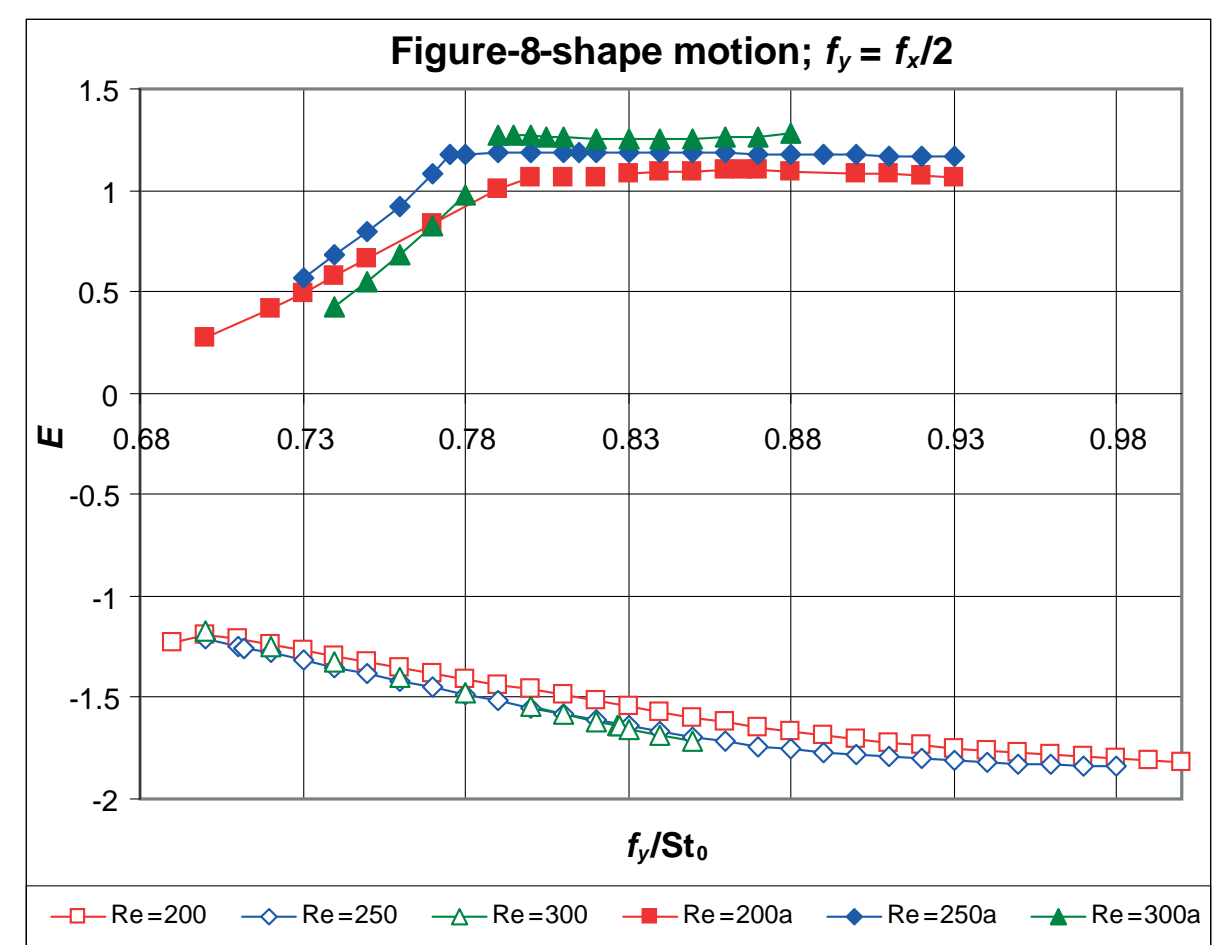

Fig. 13. Mechanical energy transfer vs. frequency ratio for clockwise $(\operatorname{Re}=200,250,300)$ and anticlockwise $(\operatorname{Re}=200 \mathrm{a}, 250 \mathrm{a}, 300 \mathrm{a})$ orbit in the upper loop

state curves - is probably due to a symmetry-breaking bifurcation (see [31]). It seems that there are two attractors in this non-linear system and the solution is attracted to one or the other depending on the parameters and initial conditions.
The rms of fixed-body lift, shown in Fig. 8, also shows different behaviour depending on direction, with the CW curves changing gradually, and the ACW exhibiting a different pattern. From around the value of $f_{y} / \mathrm{St}_{0}=0.8$, the difference between the two sets 
of curves increases with frequency ratio. There is a non-locked-in domain for ACW orbit for $\mathrm{Re}=300$ at around $f_{y} / \mathrm{St}_{0}=0.8$. This harmonizes with the observations of Gharib, who found that his 2-DoF free vibration cases were much less likely to exhibit lock-in behaviour (where the wake locks to the natural frequency of the structure for some range of parameters) [32]. Although the values are much smaller, basically the same tendencies were found for the TM and rms of torque coefficients shown in Figs. 9 and 10 (c.f. Figs. 7 and 8 ) including the locations and number of jumps.

The TM of drag is shown in Fig. 11 against the frequency ratio for the three Reynolds numbers and two directions of orbit. The notations are exactly the same as earlier. As can be seen in the figure, the values for CW orbit increase almost linearly with frequency ratio and their dependence on Reynolds number is weak, increasing only slightly with Re. We can also observe that the shapes of the ACW curves are different from that of the CW curves. The slope of the curves changes abruptly at about $f_{y} / \mathrm{St}_{0}=0.79$. Above this value the curves varys almost linearly with the frequency ratio. The TM of drag increases with Reynolds number and a stronger Re effect can be seen than for the CW cases. It is obvious from the figure that the $\mathrm{TM}$ of drag is smaller for the CW case over the large part of lock-in domain; hence it can be considered to be the more favourable orbital direction for free vibration cases.

In Fig. 12 the rms values of fixed-body drag against the frequency ratio are shown for the six cases displayed earlier. In this case the tendency is opposite compared to the TM of drag: the rms values are higher for the CW case over the whole investigated domain. This means that for CW orbit, although the TM of the drag is smaller, the oscillation amplitude of the signal is larger than for the ACW case. A relatively

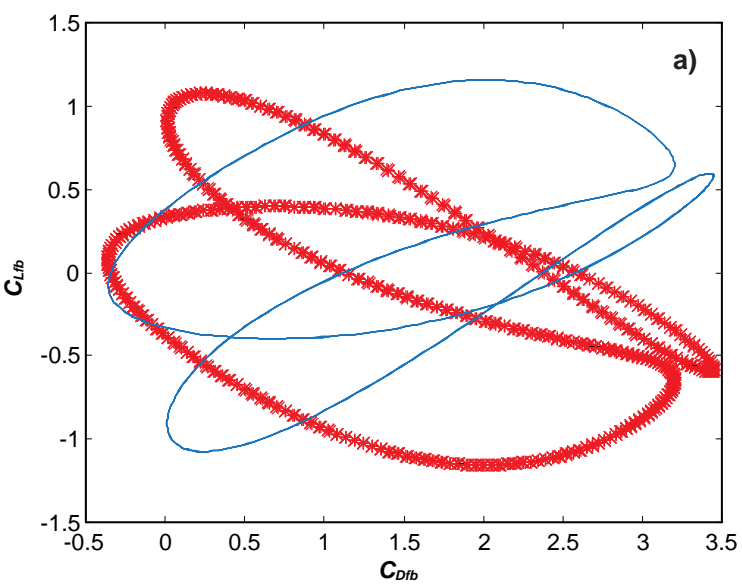

weak Re number dependency can be seen in the rms of drag (see Fig. 12).

Base pressure coefficients (not shown here) show basically the same tendency as was found for the drag for both TM and rms values for all three Reynolds numbers and both directions of orbit (see Figs. 11 and 12).

The mechanical energy transfer $E$ between the fluid and the cylinder within one motion period (based on $f_{y}$ ) is given in Fig. 13. For CW, it can be seen that $E$ curves are close to each other and always negative within the lock-in domain, while the ACW curves are always positive. These findings are in agreement with those of [18]. The positive energy transfer means that cylinder motion is amplified by the fluid, which can lead to vortex-induced vibration (VIV) of the cylinder. Hence the ACW orbit is the less favourable case from this point of view. As can be seen in the figure, $E$ is approximately constant over $f_{y} / \mathrm{St}_{0}=0.8$ for all three Re values for the ACW case.

\subsection{Pre- and post-jump analysis}

As was shown earlier, jumps were found in the TM of lift and torque for the clockwise (CW) orbit for both $\operatorname{Re}=250$ and 300 (see Figs. 7 and 9). These jumps can be attributed to vortex switches. The vicinity of a jump is investigated by different means, such as the time history of fixed body lift and drag (not shown), limit cycle curves and vorticity contours before and after the jump. Since the $\mathrm{Re}=250$ and $\mathrm{Re}=300$ results are very similar, only those for $\mathrm{Re}=250$ are shown here. The limit cycle curves $\left(C_{D f f}, C_{L f b}\right)$ can be seen in Fig. 14(a). The curves are very complex; the pre-jump curve $\left(f_{y} / \mathrm{St}_{0}=0.71213\right)$ is denoted by the thick line and the post-jump curve $\left(f_{y} / \mathrm{St}_{0}=0.71214\right)$ by the thin line. The difference in frequency ratios is only 0.00001 , but a drastic change in the outcome is

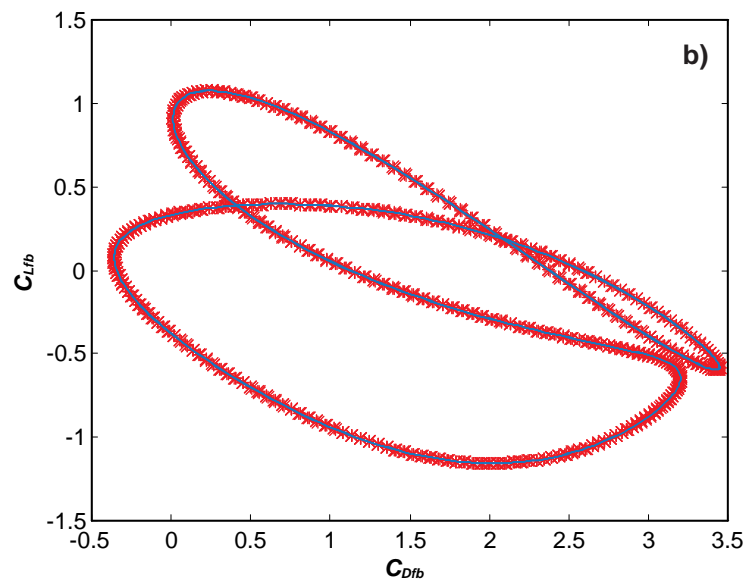

Fig. 14. Limit cycle curves, $\mathrm{Re}=250$. Thick line: $f_{y} / \mathrm{St}_{0}=0.71213$, thin line: $f_{y} / \mathrm{St}_{0}=0.71214$. a): both curves $\left(C_{D f b}, C_{L f b}\right)$; b): pre-jump curve $\left(C_{D f f}, C_{L f b}\right)$, post-jump curve $\left(C_{D f b},-C_{L f b}\right)$ 

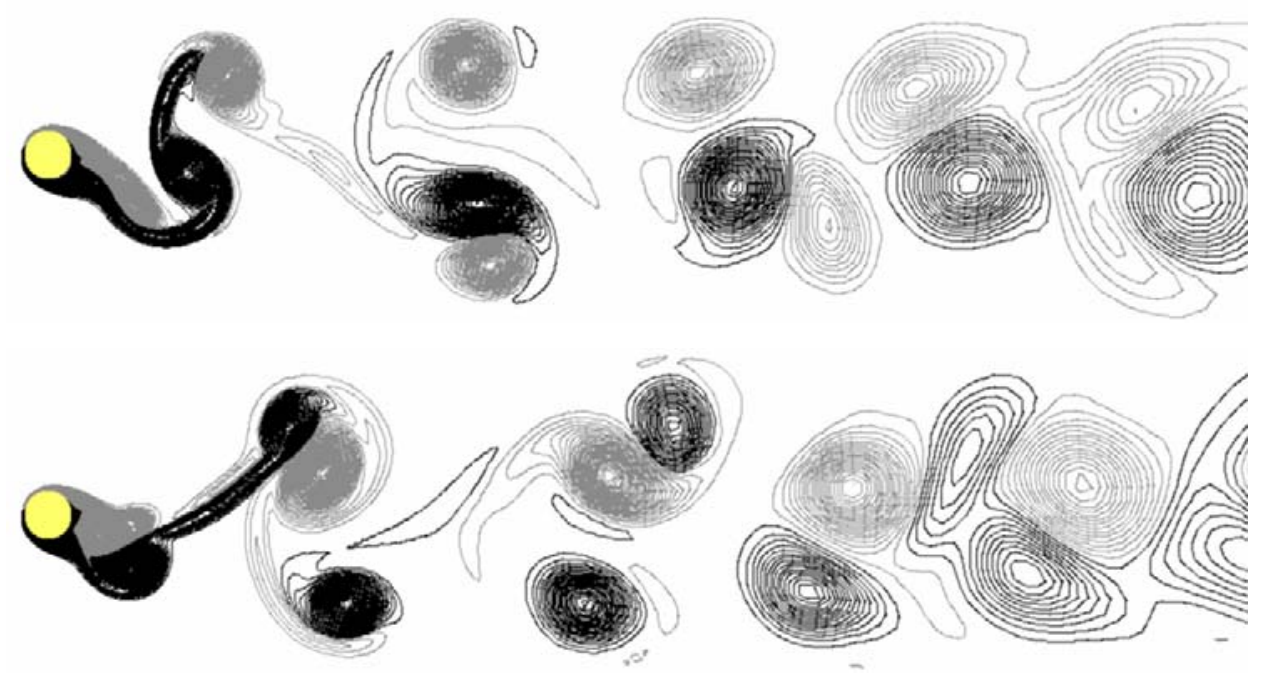

Fig. 15. Pre- and post-jump vorticity contours. Top: $f_{y} / \mathrm{St}_{0}=0.71213$, bottom: $f_{y} / \mathrm{St}_{0}=0.71214$

evident. Although it is not obvious at first sight, these curves are mirror images of each other (flipping along the line of $C_{L}=0$ ), as can also be seen in Fig. 14(b), where the post-jump $C_{L f b}$ values are replaced by $\left(-C_{L f b}\right)$ values.

The vorticity contours shown in Fig. 15 also show the drastic change of the flow patterns before and after a jump. The contours belong to the same cylinder position (at $x_{0}=y_{0}=0$; see Eqs. (5) and (6), and Fig. 2). The grey lines indicate negative vorticity values, rotating clockwise, and the black are positive, rotating anticlockwise. The vortex shedding mode starts out as $\mathrm{P}+\mathrm{S}$, meaning that a pair $(\mathrm{P})$ of vortices and a single $(\mathrm{S})$ vortex are shed in one period. It can be seen that the vortex pattern has switched to a near-mirror image: before the jump double vortices are in the lower row but then switch to the upper row. Later, however, the mode seems to shift towards $2 \mathrm{~S}$ (two single vortices are shed in one period). It is worth mentioning that the pre- and post-jump time history lift curves are not a mere reflection of each other; they are reflected and translated by a half period $(T / 2)$ with respect to each other (not shown here). That is probably the reason why the pre- and post-jump vorticity contours are not exactly mirror images of each other. It was shown earlier in [28] that for forced in-line cylinder motion the pre- and postjump time history curves, the (drag, lift) limit cycle curves and the vorticity contours are all mirror images of each other.

The present cylinder-flow system is almost reflection-symmetric about a line through the cylinder centre and parallel to the free stream velocity vector, as shown by both the limit cycle curves and vorticity contours. A physical system that is symmetric is vulnerable to symmetry-breaking bifurcation [31], and this may well be the case here.

\section{Conclusions}

Forced cylinder motion in the shape of a slender figure 8 was investigated in this numerical study using a 2D computational method based on the finite difference method. The effects of in-line amplitude $A_{x}$ and frequency ratio $f_{y} / \mathrm{St}_{0}$ on the mechanical energy transfer $E$, the time-mean (TM) and rms values of force coefficients were investigated for low-Reynolds number flows within the lock-in domain.

1) $A_{x}$ effects for clockwise (CW) orbit in the upper loop of the figure-eight for $\mathrm{Re}=150,200$ and 250:

- No jumps (vortex switches) were found,

- $E$ was always negative (hence no dangers of VIV for free vibration cases),

- TM and rms of lift and the absolute value of $E$ increase with increasing $A_{x}$ and Re,

- TM of torque coefficient tq did not show a clear tendency.

2) Frequency ratio $f_{y} / \mathrm{St}_{0}$ effects for both clockwise (CW) and anticlockwise (ACW) orbit in the upper loop of figure-eight for $\mathrm{Re}=200,250$ and 250:

a) Clockwise (CW) orbit

- $E$ was always negative (hence no dangers of VIV for free vibration cases),

- TM of drag is smaller, rms of drag is larger than for the ACW cases,

- Jumps (representing switches in vortex structures) were found in TM of lift and torque when plotted against $f_{y} / \mathrm{St}_{0}$ for $\mathrm{Re}$ $=250$ and 300; solutions on mirror-image state curves (as for in-line cylinder motion),

- Pre- and post-jump analysis showed perfect mirror-image symmetry for the (drag, lift) limit cycle curves. Vorticity contours 
are very different from each other (almost a mirror image change) before and after a jump; P+S vortex structure was found.

b) Anticlockwise (ACW) orbit:

- $E$ was always positive (dangers of VIV for free vibration cases),

- TM of lift and torque are identically zero (as for transverse cylinder motion),

- TM of drag is larger; rms of drag is smaller than for the CW cases,

- No jumps were found in the investigated domain.

Future research might include the investigation of the effect of initial conditions for the CW case in order to produce more complete state curves and confirm their symmetric nature. Closer examination of the effect of oscillation amplitude for the ACW case could also prove to be worthwhile.

\section{Acknowledgements}

The support of the Hungarian Research Foundation (OTKA Project K 76085) is gratefully acknowledged. The work was carried out as part of the TÁMOP-4.2.1.B-10/2/KONV-20100001 project in the framework of the New Hungarian Development Plan. The realisation of this project is supported by the European Union, co-financed by the European Social Fund. The author thanks Mr. L. Daróczy for designing the flow visualization software used for Fig. 15.

\section{References}

[1] Williamson, C.H.K. and Roshko, A. (1988), Vortex formation in the wake of an oscillating cylinder. Journal of Fluids and Structures 2, 355-381.

[2] Lu, X.Y. and Dalton, C. (1996), Calculation of the timing of vortex formation from an oscillating cylinder. Journal of Fluids and Structures 10, 527-541.

[3] Blackburn, H.M. and Henderson, R.D. (1999), A study of two-dimensional flow past an oscillating cylinder. Journal of Fluid Mechanics 385, 255-286.

[4] Cetiner, O. and Rockwell, D. (2001), Streamwise oscillations of a cylinder in a steady current. Part 1. Locked-on states of vortex formation and loading. Journal of Fluid Mechanics 427, 1-28.

[5] Al-Mdallal, Q.M., Lawrence, K.P. and Kocabiyik, S. (2007), Forced streamwise oscillations of a circular cylinder: Locked-on modes and resulting fluid forces. Journal of Fluids and Structures 23, 681-701.

[6] Mureithi, N.W., Huynh, K., Rodriguez, M. and Pham, A. (2010), A simple low order model of forced Karman wake. International Journal of Mechanical Sciences 52(11), 1522-1534.

[7] Kheirkhah, S. and Yarusevych, S. (2010), Two-degreeof-freedom flow-induced vibrations of a circular cylinder with a high moment of inertia ratio. Proc. ASME 2010 3rd
Joint US-European Fluids Engineering Summer Meeting and 8th Int. Conference on Nanochannels, Microchannels, and Minichannels (FEDSM-ICNM2010), Montreal, Québec, Canada, on CD ROM, pp. 1-10, Paper No. FEDSM-ICNMM2010-30042.

[8] Blevins, R.D. (1990), Flow-induced vibration. Krieger Publishing Company, Maladar.

[9] Didier, E. and Borges, A.R.J. (2007), Numerical predictions of low Reynolds number flow over an oscillating circular cylinder. Journal of Computational and Applied Mechanics 8(1), 39-55.

[10] Baranyi, L. (2008), Numerical simulation of flow around an orbiting cylinder at different ellipticity values. Journal of Fluids and Structures 24, 883-906.

[11] Stansby, P.K. and Rainey, R.C.T. (2001), On the orbital response of a rotating cylinder in a current. Journal of Fluid Mechanics 439, 87-108.

[12] Williamson, C.H.K. (2004), Vortex-induced vibrations. Annual Review of Fluid Mechanics 36, 413-455.

[13] Jeon, D. and Gharib, M. (2001), On circular cylinders undergoing two-degree-of-freedom forced motions. Journal of Fluids and Structures 15, 533-541.

[14] Jauvtis, N. and Williamson, C.H.K. (2004), The effect of two degrees of freedom on vortex-induced vibration and at low mass and damping. Journal of Fluid Mechanics 509, 23-62.

[15] Sanchis, A., Sælevik, G. and Grue, J. (2008), Two-degreeof-freedom vortex-induced vibrations of a spring-mounted rigid cylinder with low mass ratio. Journal of Fluids and Structures 24, 907-919.

[16] Baranyi, L. (2012), Computation of flow around a circular cylinder undergoing two-degree-of-freedom forced motion at low Reynolds numbers. Proc. 10th International Conference on Flow-Induced Vibration, Meskell \& Bennett (eds.), Dublin, pp. 361-368.

[17] Prasanth, T.K. and Mittal, S. (2009), Flow-induced oscillation of two circular cylinders in tandem arrangement at low Re. Journal of Fluids and Structures 25, 1029-1048.

[18] Perdikaris, P.D., Kaiktsis, L. and Triantafyllou, G.S. (2009), Computational study of flow structure and forces on a cylinder vibrating transversely and in-line to a steady stream: Effects of subharmonic forcing. Proc. ASME 2009 Pressure Vessels and Piping Conference, Symposium on Flow-Induced Vibration. Prague, on CD ROM, pp. 1-5, Paper No. PVP2009-78010.

[19] Peppa, S., Kaiktsis, L. and Triantafyllou, G.S. (2010), The effect of in-line oscillation on the forces of a cylinder vibrating in a steady flow. Proc. 7th International Symposium on Fluid-Structure Interactions, Flow-Sound Interactions, and Flow-Induced Vibration and Noise, (within FEDSM2010-ICNMM2010 ASME Conference 2010), Montreal, Québec, Canada, on CD ROM, pp. 1-8, Paper No. FEDSM-ICNMM2010-30054.

[20] Baranyi, L. (2010), Numerical simulation of the flow around a circular cylinder following a figure-8-like path. Proc. 7th International Symposium on Fluid-Structure Interactions, Flow-Sound Interactions, and Flow-Induced Vibration and Noise, (within FEDSM2010-ICNMM2010 ASME Conference 2010), Montreal, Québec, Canada, on CD ROM, pp. 1-7, Paper No. FEDSM-ICNMM2010-30888. 
[21] Harlow, F.H. and Welch, J.E. (1965), Numerical calculation of time-dependent viscous incompressible flow of fluid with free surface. Physics of Fluids 8, 2182-2189.

[22] Baranyi, L. and Shirakashi, M. (1999), Numerical solution for laminar unsteady flow about fixed and oscillating cylinders. Journal of Computer Assisted Mechanics and Engineering Sciences 6, 263-277.

[23] Baranyi, L. (2003), Computation of unsteady momentum and heat transfer from a fixed circular cylinder in laminar flow. Journal of Computational and Applied Mechanics 4, 13-25.

[24] Chakraborty, J., Verma, N. and Chhabra, R.P. (2004), Wall effects in flow past a circular cylinder in a plane channel: a numerical study. Chemical Engineering and Processing 43, 1529-1537.

[25] Kravchenko, A.G., Moin, P. and Shariff, K. (1999), BSpline method and zonal grids for simulations of complex turbulent flows. Journal of Computational Physics 151, 757-789.

[26] Daróczy, L. and Baranyi, L. (2012), Euler and secondorder Runge-Kutta methods for computation of flow around a cylinder. Proc. 26th MicroCAD International Scientific Conference, Miskolc, Hungary, Section N, pp. 1-6, Paper No. N10.

[27] Baranyi, L. (2005), Lift and drag evaluation in translating and rotating non-inertial systems. Journal of Fluids and Structures 20, 25-34.
[28] Baranyi, L. (2009), Sudden and gradual alteration of amplitude during the computation for flow around a cylinder oscillating in transverse or in-line direction. Proc. ASME 2009 Pressure Vessels and Piping Conference, Symposium on Flow-Induced Vibration. Prague, on CD ROM, pp.110, Paper No. PVP2009-77463.

[29] Baranyi, L. (2008), Effect of frequency ratio on the force coefficients of a cylinder oscillated in a uniform stream. Proc. 7th JSME-KSME Thermal and Fluids Engineering Conference, Sapporo, Japan, on CD ROM, pp.1-4, Paper No. L132.

[30] Baranyi, L., Huynh, K. and Mureithi, N.W. (2010), Dynamics of flow behind a cylinder oscillating in-line for low Reynolds numbers. Proc. 7th International Symposium on Fluid-Structure Interactions, Flow-Sound Interactions, and Flow-Induced Vibration and Noise, (within FEDSM2010-ICNMM2010 ASME Conference 2010), Montreal, Québec, Canada, on CD ROM, pp. 1-10, Paper No. FEDSM-ICNMM2010-31183.

[31] Crawford, J.D. and Knobloch, E. (1991), Symmetry and symmetry-breaking bifurcations in fluid dynamics. Annual Review of Fluid Mechanics 23, 341-387.

[32] Gharib, M.R. (1999), Vortex-induced vibration, absence of lock-in and fluid force deduction. PhD dissertation, GALCIT, California Institute of Technology, Pasadena, CA, U.S.A. 